\title{
DOS ESPECIES NUEVAS DE PARACONULARIA (SCYPHOZOA, CONULARIIDAE) EN LA FORMACIÓN LAS DELICIAS, PÉRMICO INFERIOR-MEDIO DE COAHUILA, MÉXICO
}

\author{
SARA A. QUIROZ-BARROSO, FRANCISCO SOUR-TOVAR \\ Museo de Paleontología, Departamento de Biología Evolutiva, Facultad de Ciencias, Universidad Nacional Autónoma de \\ México. Ciudad Universitaria, C.P. 04510, Cd Mex, México. \\ saqb@ciencias.unam.mx,fcosour@ciencias.unam.mx \\ JESÚS QUIROZ-BARRAGÁN \\ Museo de Paleontología de La Laguna. Avenida Juárez 580, Primero de Cobián Centro, C.P. 27000, Torreón, Coahuila. \\ paleocoah55@hotmail.com
}

\begin{abstract}
Two new species of Paraconularia (Scyphozoa, Conulariidae) from the Las Delicias Formation, lower-middle Permian of Coahuila, Mexico. Two new species Paraconularia are described from rocks of lower-middle Permian (Kungurian to Capitanian) age in the Las Delicias Formation, northeastern of Torreón, into the State of Coahuila, Mexico. These species are the first conulariids reported from the Permian of Mexico, and they have affinities with faunas of the Grandian Province from the Palaeoequatorial Realm.
\end{abstract}

Keywords: conulariids, Las Delicias Formation, Mexico, lower-middle Permian, Grandian Province.

RESUMO - São descritas duas novas espécies de conuláridos do gênero Paraconularia, provenientes das rochas do Permiano inferiormedio (Kunguriano-Capitaniano) da Formação Las Delicias, que afloram ao noreste da cidade de Torreón no Estado de Coahuila, México. Estas espécies estão relacionadas com faunas da Provincia Grandiana do Reino Paleoequatorial e representam o primeiro registro formal de conuláridos para o Permiano do México.

Palavras-chave: conuláridos, Formação Las Delicias, México, Permiano inferior-médio, Provincia Grandiana.

\section{INTRODUCCIÓN}

Los conuláridos constituyen un grupo extinto de organismos marinos, bentónicos, sésiles y ocasionalmente de hábitos gregarios. Sus fósiles son comunes en rocas de facies de aguas someras de ambientes siliciclásticos y carbonatados con edades del Neoproterozoico tardío al Triásico temprano (Ivanstov \& Fedonkin, 2002; Leme et al., 2008, 2010, 2013). Con base en comparaciones de la anatomía, morfogénesis y autoecología de los cnidarios existentes, se ha considerado que los conuláridos fueron cnidarios de la clase Scyphozoa (medusas típicas) o que estuvieron cercanamente emparentados con ellos (e.g. Kiderlen, 1937; Moore \& Harrington, 1956; Werner, 1973; Bischoff, 1978; von Salvini-Plawen, 1978; Bouillon, 1981; Van Iten, 1991a, 1992a,b; Jerre, 1994; Wade, 1994; Bergström, 1995; McKinney et al., 1995; Van Iten et al., 1996, 2000; Hughes et al., 2000; Nielsen, 2001; Collins, 2002; Leme et al., 2008, 2010). Su aspecto sería similar al de la etapa de pólipo de los escifozoos, con pólipos reducidos y tentáculos que rodeaban la región oral y un tallo que les permitía afianzarse al sustrato (Sabattini \& Hlebszevitsch, 2005). Respecto a su alimentación, se ha considerado que pudieron ser filtradores suspensívoros (Brood, 1979; Hardland \& Pickerill, 1987) o bien carnívoros, esto último por analogía con los escifozoos (Moore \& Harrington, 1956).

La diversidad de los conuláridos nunca fue alta y son escasos los géneros reconocidos para cada periodo geológico (Lucas, 2012). Durante el Ordovícico y el Devónico el grupo alcanzó su mayor diversidad, seguida por una serie de extinciones locales y sufriendo una pérdida de más del $90 \%$ de su diversidad durante la extinción masiva del Permo-Triásico (Sendino-Lara, 2009). Su extinción definitiva se ha relacionado con la revolución marina del Mesozoico, durante la cual organismos durófagos desarrollaron nuevos mecanismos para depredar el bentos epifaunal, incluídos los conuláridos (Lucas, 2012). 
En México, la presencia de conuláridos ha sido referida para rocas del Paleozoico superior. Para el Carbonífero se han descrito Conularia cf. C. subcarbonaria, Paraconularia chesterensis y Paraconularia cf. P. subulata en rocas del Misisípico (Tournaisiano-Viseano) de Oaxaca (EscalanteRuiz et al., 2014) y existen referencias al grupo para rocas del Misisípico superior-Pensilvánico inferior de Chihuahua (Bridges, 1964) y del género Paraconularia para el Pensilvánico de Oaxaca (Quiroz-Barroso \& Perrilliat, 1997; Buitrón et al., 2000; Quiroz-Barroso \& Sour-Tovar, 2006). Para el Pérmico se ha indicado la presencia del género Paraconularia en la Formación Olinalá en Guerrero (GonzálezArreola et al., 1994).

El objetivo de este trabajo es reconocer la identidad taxonómica de los conuláridos que se han encontrado en rocas pérmicas de la Formación Las Delicias, unidad que aflora en el suroeste del estado de Coahuila, en el norte de México, y establecer sus afinidades con las de otras localidades de la misma edad y así evaluar su significado paleobiogeográfico.

\section{MARCO GEOLÓGICO}

\section{Estratigrafía}

Las rocas del Pérmico que afloran en la Sierra Las Delicias fueron referidas y estudiadas inicialmente por Haarmann (1913), Haack (1914) y Böse (1921, 1923). Posteriormente se realizaron estudios más detallados de la geología del área por King (1934), King et al. (1944), Kelly (1936) y por Wardlaw et al. (1979). Con base en la bioestratigrafía de fusulínidos, braquiópodos y especialmente ammonoideos realizada por King et al. (1944), estos últimos autores dividieron la sucesión del Pérmico de las Sierra Las Delicias en cinco unidades estratigráficas informales: la basal fue llamada Las Sardinas, correspondiente al Kunguriano-Roadiano (Cisuraliano tardío), seguida por El Tordillo del KungurianoRoadiano (Cisuraliano tardío-Guadalupiano temprano), Palo Quemado del Kunguriano-Wordiano (Guadalupiano medio), La Difunta del Wordiano-Capitaniano (Guadalupiano mediotardío) y La Colorada del Capitaniano (Guadalupiano tardío). Posteriormente fueron denominadas como Formación Las Delicias por McKee et al. (1999), quienes concluyeron que los afloramientos pérmicos del suroeste de Coahuila son parte del Terreno Coahuiltecano, una unidad tectonoestratigráfica que se formó a lo largo del Misisípico-Pérmico en un arco proximal con condiciones de aguas profundas y cuya litología consiste en sucesiones volcaniclásticas intercaladas con capas de flujos de detritos calcáreos y rocas volcánicas que derivaron de este arco volcánico activo y que se acumularon por múltiples eventos de movimientos de masa. De acuerdo a los mismos autores, la influencia de actividad volcánica dio origen a volcaniclastos andesíticos y dacíticos de diversos tamaños que se estratifican con rocas clásticas ricas en carbonatos y rocas calcáreas, formando once unidades que incluyen siete litosomas con rasgos caóticos y cuatro litososmas con estratificación reconocible.

La fauna fósil fue descrita en la monografía editada por King et al. (1944), en la que se ilustra la diversidad de fusulínidos (Dunbar, 1944), braquiópodos (Cloud, 1944) y cefalópodos (Miller, 1944); trabajos posteriores incluyen el estudio de un crinoideo (Strimple, 1971), la referencia de esponjas en el área (Rigby \& Senowbari-Daryan, 1995), consideraciones taxonómicas y paleobiogeográficas de trilobites (Sour-Tovar et al., 2016) y la revisión de los braquiópodos y sus afinidades paleobiogeográficas (TorresMartínez et al., 2019).

\section{Paleobiogeografía}

Las afinidades de los braquiópodos del Pérmico de Coahuila con las faunas de Texas establecidas por Cloud (1944) y las características de las faunas de fusulínidos descritas por Dunbar (1944) fueron la base para que años más tarde, las localidades del Pérmico de Coahuila se consideraran parte de la gran Provincia Grandiana postulada por Yancey (1975), una provincia marina tropical y subtropical de aguas cálidas que durante el Pérmico temprano y medio se extendió sobre el Cratón de Norteamérica ocupando el área de Texas-Nuevo México-Coahuila. Esta provincia se distinguió de las otras dos provincias bióticas marinas que también se desarrollaron sobre el Cratón de Norteamérica: la Provincia Boreal de aguas frías que abarcó la actual región Ártica, y la Provincia Cordillerana de aguas templadas que ocupó gran parte del actual territorio de Estados Unidos y la región adyacente de Canadá (Yancey, 1975). Las biotas de la Provincia Grandiana presentan una alta diversidad en la que sobresalen especies de macroforaminíferos, corales compuestos, esponjas y algas, así como braquiópodos y moluscos ornamentados; entre los braquiópodos destacan lytónidos y richthofénidos, formas altamente modificadas o aberrantes.

En los últimos años se ha reconocido la afinidad de otras faunas marinas del Pérmico temprano y medio del centro y sur de México con la fauna de la Provincia Grandiana (SourTovar \& Quiroz-Barroso, 2015); la diversidad biológica que se encuentra en estas localidades demuestra la heterogeneidad ambiental que presentó este océano, así como los cambios geográficos que se dieron en toda la región y que se asocian principalmente al acercamiento de Gondwana-Laurencia y al cierre del océano que separaba a estos grandes continentes (Rosales- Lagarde et al., 2005; Sour-Tovar \& Quiroz-Barroso, 2015; Sour-Tovar et al., 2016).

\section{MATERIAL Y MÉTODOS}

Las localidades fosilíferas de la Sierra Las Delicias son consideradas como las más representativas del Pérmico de Coahuila. Prospecciones recientes, realizadas principalmente por Jesús Quiroz, coautor de este trabajo, han derivado en el hallazgo de once ejemplares de conuláridos. El material estudiado proviene de tres localidades que se ubican al noreste de la ciudad de Torreón (Figura 1): Cañón San Francisco, en donde aflora la base de la sucesión pérmica de la Formación Las Delicias descrita por King et al. (1944) que se relaciona con la parte superior de la unidad informal Las Sardinas propuesta por Wardlaw et al. (1979) y que corresponde a La Cría, uno de los litosomas estratificados estudiados por 
McKee et al. (1999) que se mencionan en el marco geológico del presente trabajo; las otras dos localidades son Cerro San Pedro y Cerro Prieto, en las que aflora la parte superior de la Formación Las Delicias descrita por King et al. (1944) que se relaciona con la parte superior de la unidad informal La Difunta propuesta por Wardlaw et al. (1979) (Figura 2) y que también corresponde al litosoma La Cría.

Loc. 1. Noreste de Cerro Prieto, en las coordenadas $26^{\circ} 25^{\prime} \mathrm{N}$ y $102^{\circ} 56^{\prime} \mathrm{O}$ (zona de Timorites). Wordiano-Capitaniano. Fósiles característicos, Timorites sp., Polydiexodina shumardi y Polydiexodina capitanensis. Capas 4 a 7 de la sección Difunta de King et al. (1944), lutita negra con concreciones, alternada con capas de caliza. Caliza lenticular de la capa 2 en la Sección Malascachas propuesta por King et al. (1944).

Loc. 2. Cerro San Pedro, en las coordenadas $26^{\circ} 22^{\prime} \mathrm{N}$ y $102^{\circ} 55^{\prime} \mathrm{O}$ (zona Timorites). Wordiano-Capitaniano. Fósiles característicos, Timorites sp., Polydiexodina shumardi y $P$. capitanensis. Capas 4 a 7 de la sección Difunta de King et al. (1944), lutita negra con concreciones, alternada con capas de caliza. Caliza lenticular de la capa 2 en la Sección Malascachas de King et al. (1944).

Loc. 3. Cañón San Francisco, en las coordenadas $26^{\circ} 18^{\prime} \mathrm{N}$ y $102^{\circ} 52^{\prime} \mathrm{O}$ (zona de Perrinites). Leonardiano. Kunguriano-Roadiano. Fósiles característicos, Perrinites hilli en concreciones dentro de lutita. Capas 25 y 26 de la Sección Difunta de King et al. (1944). Capas 18 a 20 de la sección Malascachas de King et al. (1944).

Para el estudio taxonómico se siguió la clasificación de Leme et al. (2004) y los términos morfológicos utilizados por Sinclair (1940, 1942, 1948, 1952), Moore \& Harrington (1956), Bischoff (1978), Van Iten (1991a,b), Van Iten et al. (1996) y Leme et al. (2004).

\section{PALEONTOLOGÍA SISTEMÁTICA}

El material referido en este trabajo está depositado en el Museo Paleontológico de La Laguna, en la Ciudad de Torreón, Coahuila, México (MPLL) y en el Museo de Paleontología de la Facultad de Ciencias, Universidad Nacional Autónoma de México (FCMP).

Phylum CNIDARIA Hatscheck, 1888

Clase SCYPHOZOA Götte, 1887

Subclase CONULATA Collins, 2002

Orden CONULARIIDA Miller \& Gurley, 1896

Familia CONULARIIDAE Walcott, 1886

\section{Paraconularia Sinclair, 1940}

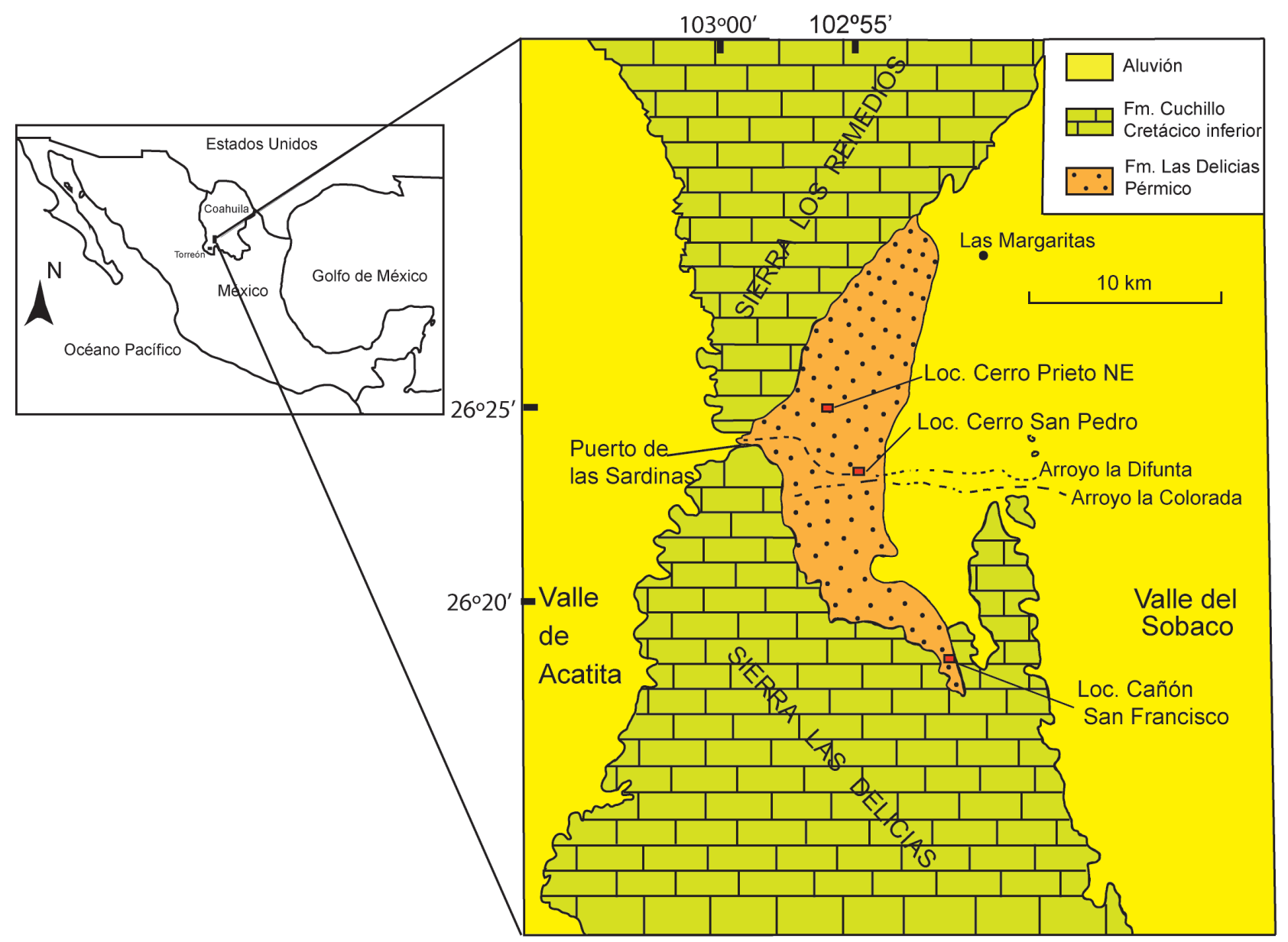

Figura 1. Afloramientos pérmicos de la Formación Las Delicias, Coahuila, México. Ubicación de las localidades de donde provienen los conuláridos estudiados.

Figure 1. Permian outcrops of the Las Delicias Formation, Coahuila, Mexico. Location of the localities from which the studied conulariids come. 
Especie tipo. Conularia inaequicostata de Koninck, 1883. Designación subsecuente por Sinclair (1940) del Carbonífero de Bélgica.

Diagnosis. Costillas transversales fuertes, que en la línea media cambian de dirección, alternando o coincidiendo. Ángulo apical pequeño, menor de $24^{\circ}$, a excepción de Paraconularia africana (Sharpe, 1856), con $36^{\circ}$. Surco angular profundo y ancho, ornamentado con costillas transversales que se interrumpen, alternando. Costillas transversales pueden portar tubérculos. Tipo de conexión arco gótico, e incluso arco gótico flexionado y curva angulosa (enmendada por Sendino-Lara, 2009).

Distribución estratigráfica. Desde el Ordovícico Medio al Triásico Tardío (Sendino-Lara, 2009).

Distribución geográfica. En los paleocontinentes: Avalonia, Báltica, sur de China, Gondwana (regiones Antártida, Andes Centrales, Armórica, Australia, Cordillera Oriental, Cuenca Paraná, Escudo Brasil, Hoggar, India, Occidentalia, Sudáfrica y Turingia), Kazajstán, Laurencia y Siberia (Sendino-Lara, 2009).

\section{Paraconularia kingii sp. nov.}

(Figuras 3A-G)

Diagnosis. Exoskeleton with transverse ribs of the gothic arch type, continuous in the midline, fine, in number from 11 to 24 per $10 \mathrm{~mm}$; angular groove present.

Diagnosis. Exoesqueleto con costillas transversales del tipo arco gótico, continuas en la línea media, finas, en número de 11 a 24 por cada $10 \mathrm{~mm}$; surco angular presente.

Descripción. Concha de 23 a $26 \mathrm{~mm}$ de longitud preservada, no se observan la abertura ni el ápice. Anchura varía de 9 a $24 \mathrm{~mm}$ en los especímenes estudiados. Superficie facial con costillas transversales finas de tipo arco gótico, de 11 a 24 por cada $10 \mathrm{~mm}$. La línea media está débilmente indicada por el cambio de dirección de las costillas, pero sin representar interrupción de éstas. Costillas con tubérculos escasamente visibles, que en el espécimen mejor conservado son siete por cada mm, separados entre sí por espacios menores a su ancho. Aristas recorridas longitudinalmente por un surco angular angosto y poco profundo, con costillas transversales coalescentes. Las medidas están en la Tabla 1.

Material examinado. Holotipo: FCMP-1258. Paratipos: MPLL-9 (Plastotipo FCMP-1259); MPLL-10 (Plastotipo FCMP-1260); MPLL-11; MPLL-12; MPLL-13; FCMP-1261. Comentarios. Paraconularia kingii presenta similitud con la especie P. leonardensis (Finks, 1955) descrita para las formaciones Cathedral Mountain, (Kunguriano) y Cherry Canyon (Roadiano) del oeste de Texas, en Estados Unidos (Finks, 1955; Van Iten, 1991c) por presentar conchas pequeñas, sección transversal cuadrada y costillas en arco gótico, pero difiere en que la especie de Texas tiene exoesqueleto muy angosto, con un una anchura máxima de $5 \mathrm{~mm}$, así como una línea media con costillas transversales usualmente alternadas y una línea media muy marcada. Un ejemplar proveniente de la Formación Olinalá, del Pérmico Medio de Guerrero,

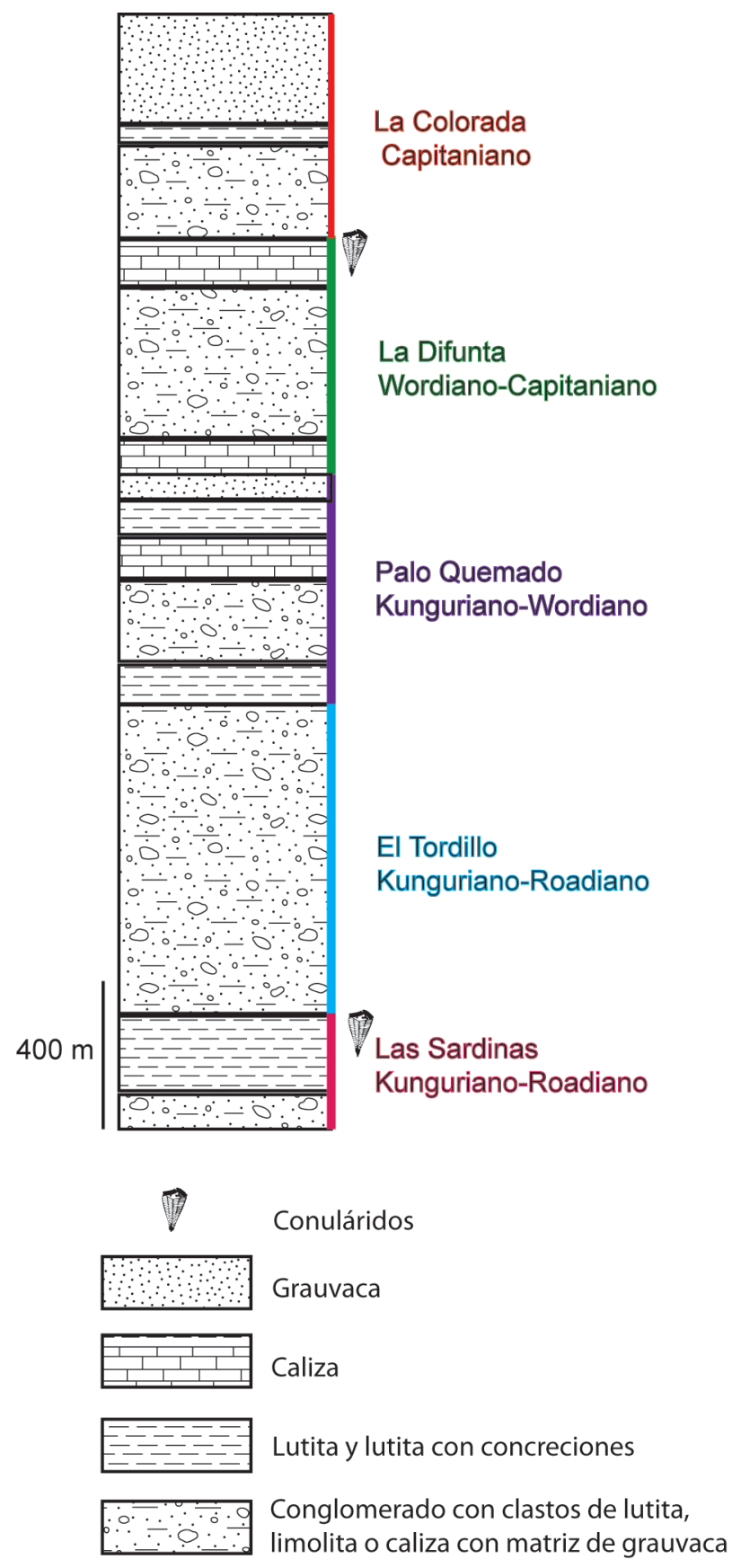

Figura 2. Sección estratigráfica del Pérmico de Las Delicias; se señalan las capas portadoras de conuláridos estudiados en este trabajo. Unidades propuestas por Wardlaw et al. (1979).

Figure 2. Stratigraphic section of the Permian of Las Delicias; beds with the conulariids studied in this work are indicated. Units proposed by Wardlaw et al. (1979).

México fue comparado con esta especie por González-Arreola et al. (1994).

Etimología. En honor a la memoria de Robert E. King, como un reconocimiento a su importante aporte para el estudio de la paleontología y la estratigrafía del Pérmico de Coahuila, norte de México.

Localidades. Cerro San Pedro y noreste de Cerro Prieto. Wordiano-Capitaniano, zona de Timorites. 

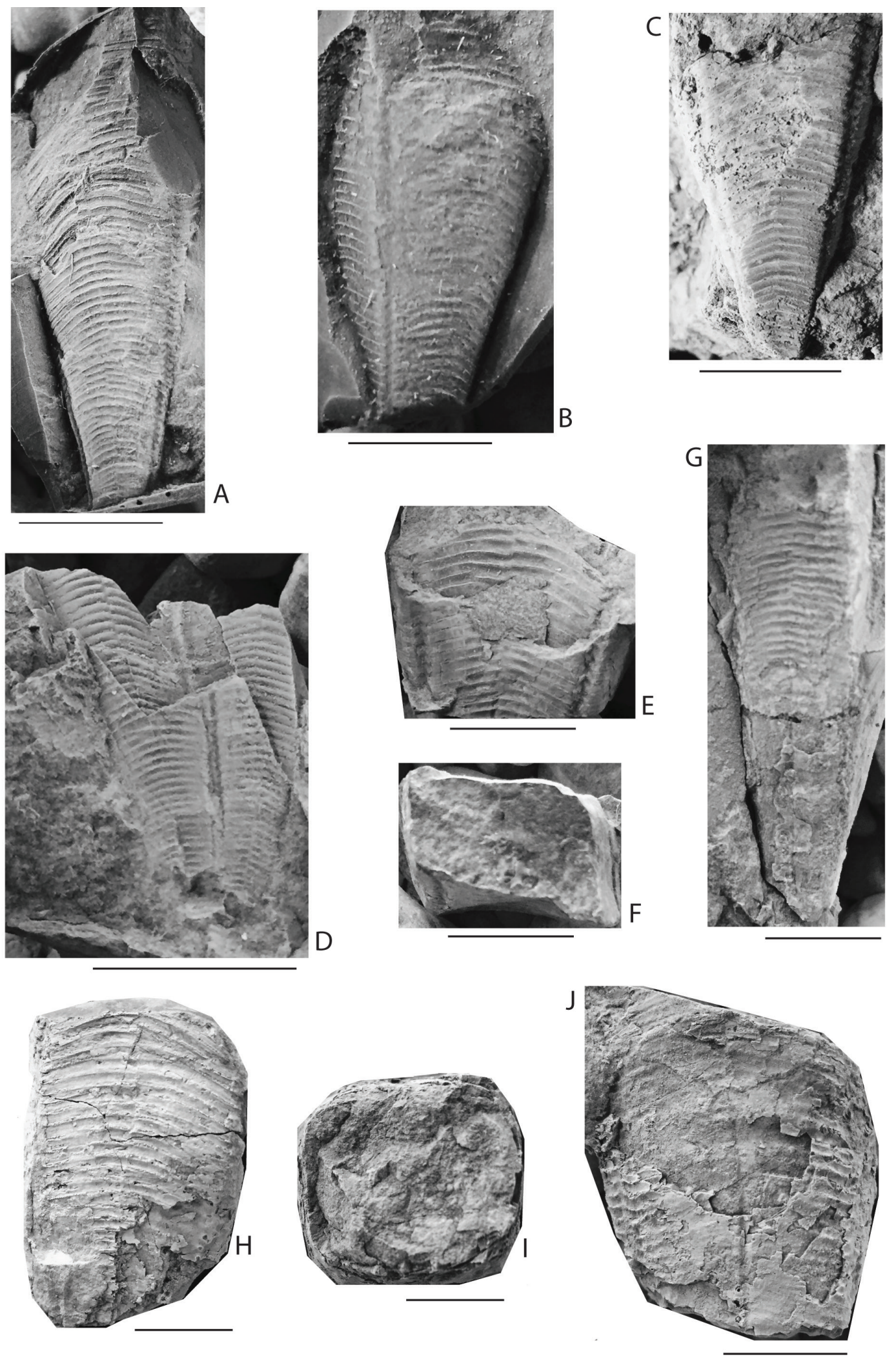

Figura 3. A-G, Paraconularia kingii sp. nov.: A, molde de silicón, vista frontal, FCMP-1259; B, molde de silicón, vista lateral, FCMP-1260; C, vista frontal, FCMP-1258; D, fragmento, vista frontal y de otras caras, MPLL-11; E, fragmento, vista frontal, MPLL-12; F, corte transversal deformado, MPLL-12; G, vista frontal, MPLL-13. H-J, Paraconularia kikapu sp. nov.: H, fragmento, vista frontal, I, corte transversal, MPLL-14; J, fragmento, vista frontal, MPLL15. Escalas $=10 \mathrm{~mm}$.

Figure 3. A-G, Paraconularia kingii sp. nov.: A, silicone mold, frontal view, FCMP-1259; B, silicone mold, lateral view, FCMP-1260; C, frontal view, FCMP1258; D, fragment, frontal view and another faces, MPLL-11; E, fragment, frontal view, MPLL-12; F, cross-section, deformated, MPLL-12; G, frontal view, MPLL-13. H-J, Paraconularia kikapu sp. nov.: H, fragment, frontal view, I, cross-section, MPLL-14; J, fragment, frontal view, MPLL-15. Scale bars = 10 mm. 
Tabla 1. Medidas de los ejemplares de la especie Paraconularia kingii sp. nov. (en mm), excepto *.

Table 1. Measurements of specimens of Paraconularia kingii sp. nov. (in mm), except*.

\begin{tabular}{|c|c|c|c|c|c|}
\hline Ejemplar & Cost. transv./10 mm* & Número de tubérculos/mm* & Longitud preservada & Anchura & Espacio \\
\hline MPLL-9 & 14 & - & 23 & 12 & 1 \\
\hline MPLL-10 & 16 & - & 21 & 9 & 0.8 \\
\hline FCMP-1258 & 15 & $7 ?$ & 19 & 10 & 1 \\
\hline MPLL-11 & 16 & - & 18 & 9 & 0.4 \\
\hline MPLL-12 & 11 & - & 11 & 12 & 0.7 \\
\hline MPLL-13 & $11-12$ & - & 7 & 24 & 0.1 \\
\hline FCMP-1261 & 24 & - & 26 & 9 & 0.4 \\
\hline
\end{tabular}

Paraconularia kikapu sp. nov.

(Figuras 3H-J, 4A-F)

Diagnosis. Exoskeleton with thick and spaced transverse ribs of the gothic arch type, in number from seven to ten per $10 \mathrm{~mm}$, which are intertwined in the wide and deep angular grooves. Diagnosis. Exoesqueleto con costillas transversales gruesas y espaciadas del tipo arco gótico, en número de siete a diez por cada $10 \mathrm{~mm}$, que se entrelazan en los surcos angulares anchos y profundos.

Descripción. Concha de tamaño mayor a los $34 \mathrm{~mm}$, aunque de longitud total indeterminada por tratarse de fragmentos, por lo que no se observan la abertura y el ápice. Anchura de 13 a $26 \mathrm{~mm}$ en los especímenes estudiados. Sección transversal cuadrada. Superficie facial con costillas transversales gruesas de tipo arco gótico, que aumentan en número hacia el extremo apical, separadas entre sí por surcos intercostales de ancho igual o menor al de las costillas. Líneas medias débilmente indicadas por el cambio de dirección de las costillas, pero sin marcarse interrupción de éstas. Tubérculos presentes (cuatro por $\mathrm{mm}$ ), con crestas intercostales mal conservadas. Aristas redondeadas, recorridas longitudinalmente por un surco angular fuertemente deprimido, en donde se entrelazan las costillas transversales, coalescentes. Las medidas están en la Tabla 2.

Material examinado. Holotipo: FCMP-1262. Paratipos: MPLL-14; MPLL-15; MPLL-16.

Comentarios. Paraconularia kikapu sp. nov. se diferencía de $P$. kingii sp. nov. por su mayor tamaño, costillas transversales menos numerosas, más grandes y espaciadas y por el entrelazado de las costillas en el surco angular. Muestra similitud con Diconularia meadepeakensis Weldon \& Shi, 2003 de la Formación Phosphoria, Miembro Meade Peak (Roadiano), Idaho, USA, por presentar un número similar de costillas transversales (7-8 en $10 \mathrm{~mm}$ ), sin embargo esta última especie se caracteriza por tener un menor tamaño (27 mm de longitud) y una sección transversal romboidal, además de otras características del género como tubérculos subcirculares prominentes y aristas simples.

Especies de Paraconularia del Paleozoico Superior con las costillas entrelazadas en el surco angular son: $P$. quadrisulcata
(Sowerby, 1821) del Carbonífero (Pensilvánico mediosuperior) de Salop, Inglaterra, sin embargo difiere en que puede tener pequeños tubérculos sobre costillas transversales y más raramente crestas intercostales adaberturales, así como una terminación adabertural lobulada; $P$. acutilirata Fletcher, 1938 del Guadalupiano de Nueva Gales del Sur (Australia), presenta un ángulo apical de unos $26^{\circ}$, caras cóncavas en la región apical y planas o ligeramente convexas en la región abertural, una línea media marcada por interrupción de las costillas transversales que suelen ser coalescentes y raramente alternando en dicha línea sin llegar a atravesarla. Otra especie, Paraconularia derwentensis (Johnston, 1887) del Pérmico (Kunguriano) de Gondwana (Australia, Nueva Zelanda y Tasmania) presenta un surco angular amplio y profundo, ornamentado por costillas transversales alternantes, entrelazándose entre ellas en las aristas; sin embargo, las costillas transversales son finas, con una densidad media de 5 en $5 \mathrm{~mm}$.

Paraconularia suarezriglosi Babcock, 1988, del Pérmico (Sakmariano a Artinskiano) de Bolivia, es una especie moderadamente angosta con costillas transversales recurvadas y ampliamente espaciadas que usualmente alternan en la línea media, sin embargo difiere de $P$. kikapu sp. nov. por presentar un gran tamaño (aproximadamente $17 \mathrm{~cm}$ ), una conexión de la costillas transversales de tipo curva flexionada, un ángulo apical mayor $\left(22^{\circ}\right)$ y ausencia de los tubérculos y crestas intercostales presentes en la especie de la Formación Las Delicias.

Paraconularia chesterensis (Worthen, 1883) del Misisípico de Alabama, Kentucky, Illinois, Indiana, Iowa, Missouri, Nevada, presenta cierta semejanza con P. kikapu sp. nov. por presentar costillas transversales espaciadas, con 4-5 tubérculos por mm, presencia ocasional de espinas adaperturales y costillas entrelazadas en el surco angular, pero su tamaño es mayor (más de $20 \mathrm{~cm}$. de longitud) y las costillas transversales son de tipo curva flexionadas, sin espinas adapicales.

Etimología. El nombre de la especie hace referencia al pueblo originario kikapú que habita en el Estado de Coahuila.

Localidad. Cañón San Francisco. Kunguriano-Roadiano, zona de Perrinites. 

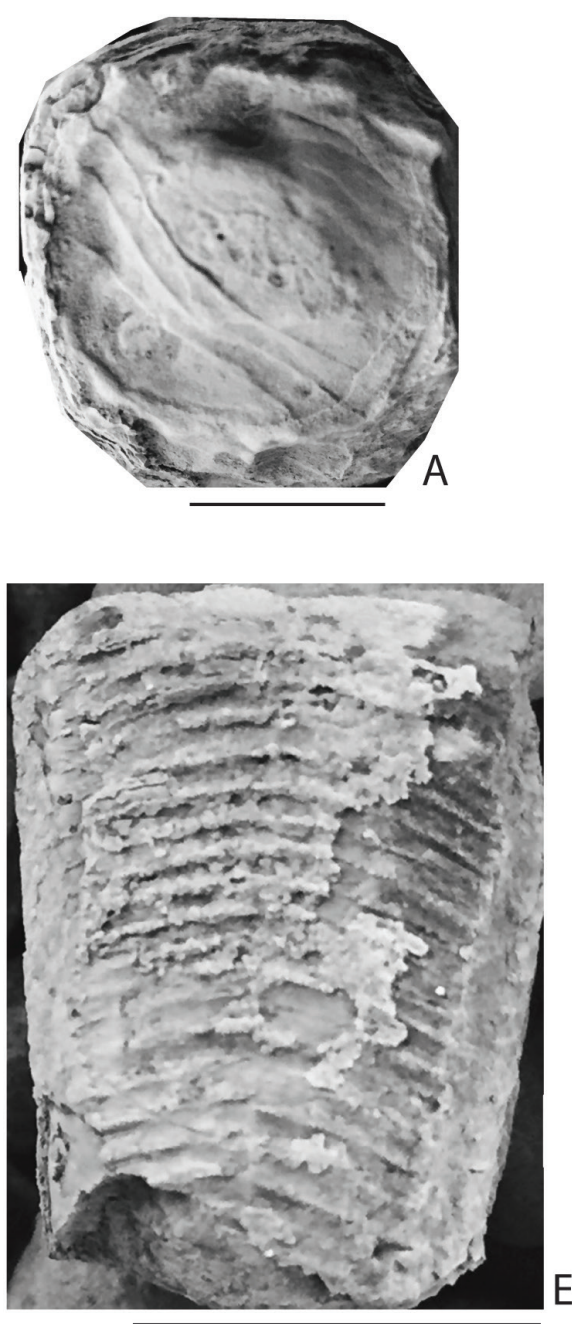
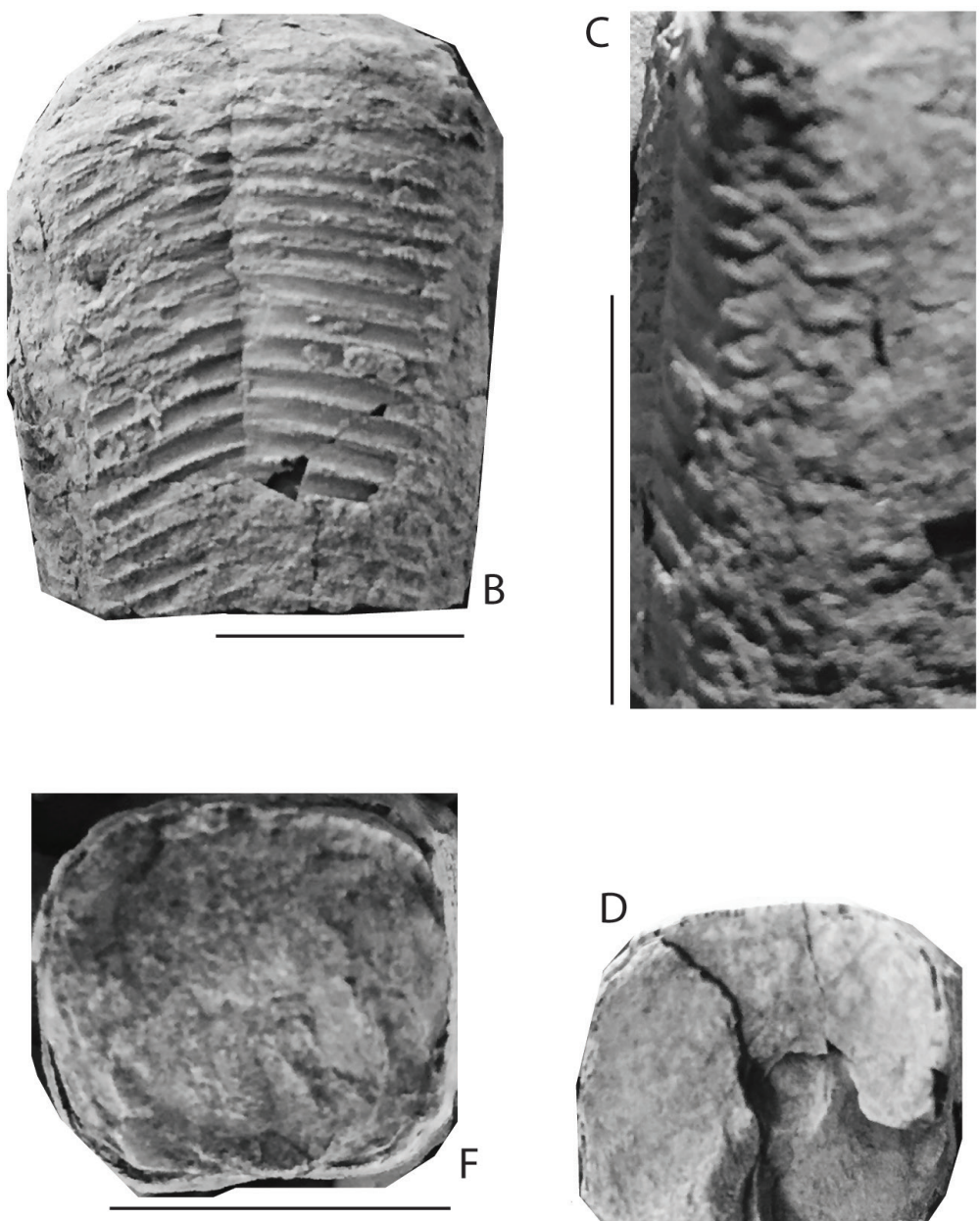

Figura 4. A-F, Paraconularia kikapu sp. nov.: A, corte transversal, MPLL-15; B, vista frontal, FCMP-1262; C, detalle de la arista con el surco longitudinal y las costillas transversales entrelazadas, FCMP-1262; D, corte transversal, FCMP-1262; E, vista frontal, MPLL-16; F, corte transversal, MPLL-16. Escalas $=10 \mathrm{~mm}$.

Figure 4. A-F, Paraconularia kikapu sp. nov.: A, cross-section, MPLL-15; B, frontal view, FCMP-1262; C, detail of the groove with the longitudinal ridge and the criss crossed transverse ribs, FCMP-1262; D, cross-section, FCMP-1262; E, frontal view, MPLL-16; F, cross-section, MPLL-16. Scale bars $=10$ mm.

Tabla 2. Medidas de los ejemplares de la especie Paraconularia kingii sp. nov. (en mm), excepto *.

Table 2. Measurements of specimens of Paraconularia kingii sp. nov. (in mm), except*.

\begin{tabular}{lccccc}
\hline Ejemplar & Cost. transv./10 mm* & Número de tubérculos $/ \mathrm{mm}^{*}$ & Longitud preservada & Anchura & Espacio \\
\hline MPLL-14 & $7-8$ & 4 & 34 & 22 & 26 \\
MPLL-15 & 7 & - & 28 & 1.3 & 1.3 \\
FCMP-1262 & $7-8$ & 4 & 24 & 13 & 1.3 \\
MPLL-16 & $9-10$ & 4 & 9 & 1.5 \\
\hline
\end{tabular}

\section{DISCUSIÓN}

\section{Consideraciones tafonómicas}

Los conuláridos de la Formación Las Delicias que se han encontrado son escasos. Algunos ejemplares se encuentran fragmentados y desgastados en su superficie, indicando la remoción de las conchas antes del sepultamiento final. Otros ejemplares completos se encuentran incluidos en concreciones calcáreas formadas durante la diagénesis temprana, lo que favoreció su preservación.

\section{Consideraciones biogeográficas}

El Pérmico presentó un marcado provincialismo marino, especialmente durante el Pérmico temprano. Con base en la distribución de braquiópodos, Waterhouse \& BohamCarter (1975), Grunt \& Shi (1997), Shi \& Grunt (2000) y Shen et al. (2009) reconocieron tres reinos para ese tiempo: Gondwánico, Boreal y Paleoecuatorial (Weldon \& Shi, 2003). Los géneros de conuláridos que han sido reconocidos hasta ahora para el Pérmico incluyen a Notoconularia Thomas, 1969, Gondaconularia Waterhouse, 1986, Cheliconularia Waterhouse, 
1986, Neoconularia Sugiyama 1942, Calloconularia Sinclair 1952, Dinoconularia Sinclair 1952, Paraconularia Sinclair, 1940, Mesoconularia Boucek, 1939 y Conularia Sowerby, 1821; de este último varios ejemplares fueron reasignados al género Paraconularia. Estos géneros han sido encontrados anteriormente en el Pérmico de Australia, India, Nueva Zelanda, Pakistán, Irán, Afganistán, Kashmir, China, Japón, Rusia, Alemania, Canadá, Estados Unidos de América y Bolivia (Weldon \& Shi, 2003). Waterhouse (1979) señaló que la distribución paleobiogeográfica de los conuláridos pérmicos sugiere que tuvieron una marcada preferencia para habitar regiones de aguas frescas-frías, ya que la diversidad es más alta en las regiones polares que en la región paleoecuatorial, como se puede apreciar al revisar la distribución de los géneros en las distintas provincias y reinos (Tabla 3). Debido a lo anterior, los conuláridos han sido considerados buenos indicadores de patrones de migración, aportando información a la comprensión de la configuración paleogeográfica y del movimiento de terrenos tectónicos (Weldon \& Shi, 2003).

Paraconularia fue un género de distribución estratigráfica amplia (Silúrico Medio a Triásico) de manera global. A diferencia del Pérmico temprano en que muestra una distribución en las regiones polares (reinos Boreal y Gondwánico), durante el Pérmico medio y tardío refleja posibles rutas de migración hacia zonas transicionales (oeste de Texas, USA., dentro de la Provincia Grandiana; Provincia Sino-mongol-japonesa en el este de Asia; Provincia Iranian de Iran; y también al sur de China, Alemania y Rusia). Weldon \& Shi (2003) han considerado posibles migraciones desde el Reino Boreal hacia aguas templadas del mar Zechstein en dirección suroeste, a lo largo de la plataforma continental y la plataforma rusa y de ahí hacia el sur en una corriente de agua fría "de tipo californiana" hacia el oeste de Texas, así como de las regiones polares hacia la Provincia Sino-mongoljaponesa. Rutas de migración similares han sido propuestas para el braquiópodo Yakovlevia del Reino Boreal (Shi, 1995). En el hemisferio sur posiblemente la migración también tuvo lugar a través de los márgenes continentales de Gondwana mediante island hopping y movimientos de terrenos en el Tethys, y también a través de corrientes de agua profundas y frías que se desplazaban en dirección norte a través del Tethys; estas rutas son comparables con las señaladas por Shi \& Grant (2000), las cuales permitieron intercambios bióticos de braquiópodos entre los reinos Gondwánico y Boreal (Weldon \& Shi, 2003).

De acuerdo a lo anterior, a las relaciones tectónicas del área de Texas-Nuevo México-Coahuila establecidas en estudios previos (Rosales-Lagarde et al., 2005) y a la similitud de sus faunas (King et al., 1944), las dos especies nuevas de Paraconularia encontradas en rocas del Pérmico medio de la Formación Las Delicias están relacionadas con la Provincia Grandiana, dentro del Reino Paleoecuatorial (Figura 5).

\section{CONCLUSIONES}

Las dos especies de conuláridos provenientes de la Formación Las Delicias representan especies nuevas del género Paraconularia y el primer registro formal de conuláridos para el Pérmico de México. P. kingii sp. nov. proviene de rocas superiores de la Formación Las Delicias, correspondientes a la zona de Timorites (en el sentido de King, 1944), de edad Wordiano-Capitaniano. P. kikapu sp. nov. proviene de rocas basales de la misma formación, en la zona de Perrinites, de edad Kunguriano-Roadiano. Ambas especies son parte de las faunas de la Provincia Grandiana del Reino Paleoecuatorial que abarcó la región de Texas-Nuevo México-Coahuila durante el Guadalupiano (Roadiano-Capitaniano), resultado de migraciones a través de corrientes marinas de aguas frías, a partir de la paleoprovincia Boreal hacia la zona ecuatorial.

\section{AGRADECIMIENTOS}

Los autores agradecen a H. Van Iten del Hanover College, Indiana, a un árbitro anónimo y a J. Leme, editora adjunta de la Revista Brasileira de Paleontologia por sus valiosas

Tabla 3. Distribución paleobiogeográfica de los géneros pérmicos de conuláridos, de acuerdo a Weldon y Shi (2003).

Table 3. Paleobiogeographic distribution of the Permian conulariids genera, according to Weldon and Shi (2003).

\begin{tabular}{|c|c|c|}
\hline Género & Distribución geográfica & Provincias y Reinos \\
\hline Cheliconularia & endémico & Provincia Himalaya del Reino Gondwánico \\
\hline Gondaconularia & endémico & Provincia Austrazeana del Reino Gondwánico \\
\hline Neoconularia & endémico & Provincia Chino-Mongol-Japonesa del Reino Boreal \\
\hline Calloconularia & bi-templado & $\begin{array}{l}\text { Provincia Himalaya, del Reino Gondwánico con migraciones } \\
\text { hacia la Provincia Chino-Mongol-Japonesa durante el Cisuraliano } \\
\text { y Guadalupiano temprano }\end{array}$ \\
\hline Notoconularia & endémico & Provincia Austrazeana del Reino Gondwánico \\
\hline Paraconularia & bi-templado & cosmopolita \\
\hline Diconularia & endémico & Provincia Cordillerana del Reino Paleoecuatorial \\
\hline Mesoconularia & endémico & Reino Boreal \\
\hline Conularia & bi-templado & $\begin{array}{l}\text { Reino Boreal y posiblemente también ocurrió en la Provincia } \\
\text { Himalaya del Reino Gondwánico }\end{array}$ \\
\hline
\end{tabular}




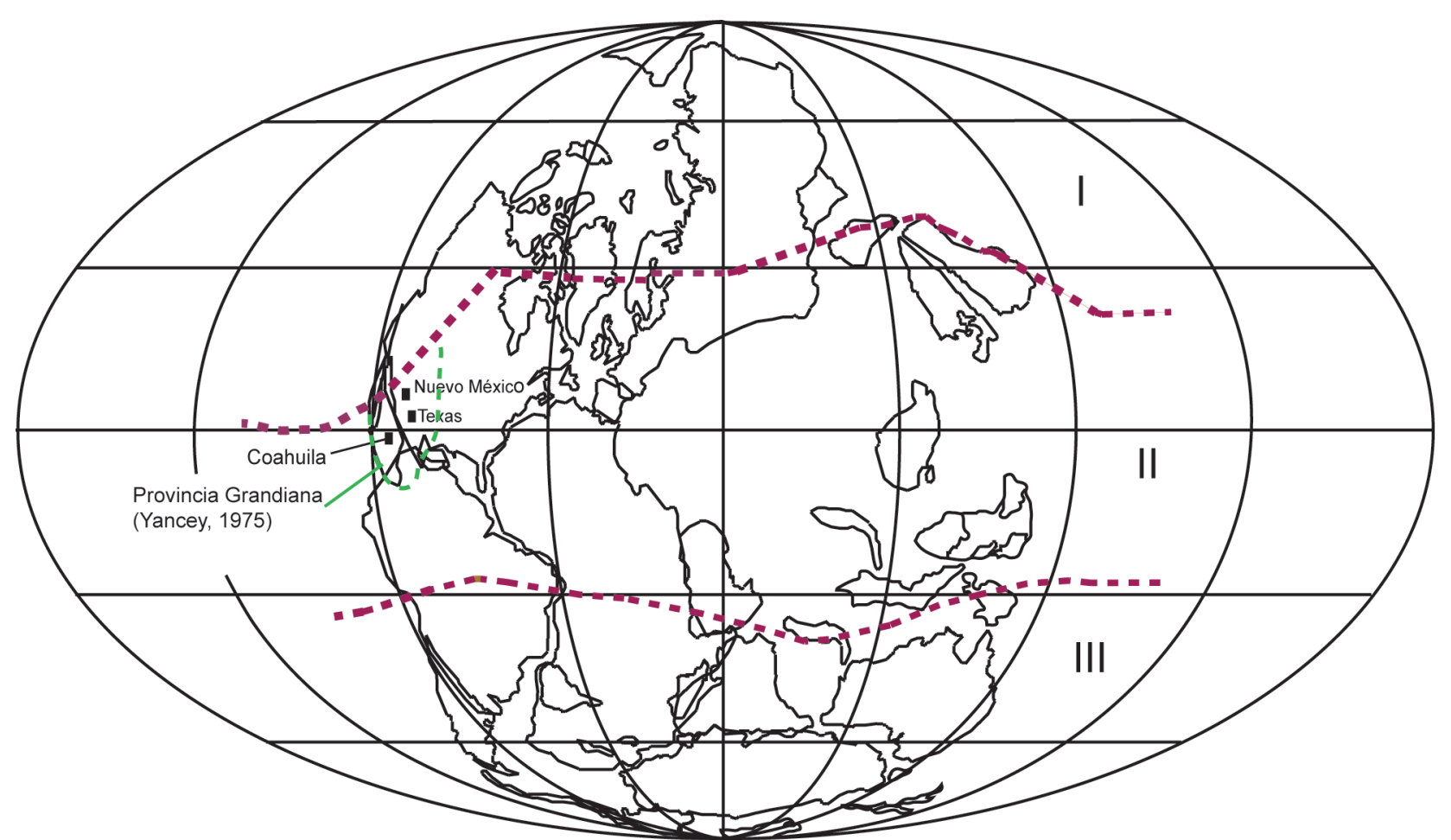

Figura 5. Distribución paleogeográfica de las especies estudiadas, durante el Pérmico medio. Reinos biogeográficos: I Boreal, II Paleoecuatorial, III Gondwánico y extensión de la Provincia Grandiana (Yancey, 1975) de acuerdo a Shen et al. (2009). Mapa base modificado de Ziegler et al. (1997).

Figure 5. Paleogeographic location of the species studied during the middle Permian. Biogeographic realms: I Boreal, II Paleoequatorial, III Gondwanic, and extension of the Grandiana Province (Yancey, 1975) according to Shen et al. (2009). Base map modified from Ziegler et al. (1997).

observaciones al manuscrito original; a D. Navarro-Santillán, L. Martin-Medrano y R. Villanueva-Olea por su apoyo técnico en la elaboración de las figuras y de los moldes de silicón.

\section{REFERENCIAS}

Babcock, L.E. 1988. New Permian conulariid from Bolivia. Journal of Paleontology, 62:617-619.

Bergström, J. 1995. Conulariid affinities: a discusión. Geologiska Föreningens i Stockholm Förhandlingar, 117:245-246.

Bischoff, G.C.O. 1978. Internal structures of conulariid test and their functional significance, with special reference to Circonulariina n. suborden (Cnidaria, Scyphozoa). Senckenbergiana Lethaea, 59:275-327.

Böse, E. 1921. On the Permian of Coahuila, northern Mexico. American Journal of Science, 1:187-194.

Böse, E. 1923. Vestiges of an ancient continente in northeast Mexico. American Journal of Science, 6:127-136, 194-214, 310-337.

Boucek, B. 1939. Conularida. In: O.H. Schindewolf(ed.) Handbuch der Paläozoologie, Verlag von Gerbruder Borntraeger, p. 113-131.

Bouillon, J. 1981. Origine et phylogenèse des cnidaries et des hydropolypeshydroméduses. Annales de la Société Royale Zoologique de Belgique, 3:45-56.

Bridges, L.W. 1964. Geology of Mina Plomosas-Placer de Guadalupe area, Chihuahua, Mexico. Midland, West Texas Geological Society, 112 p. (Publication 64-50).
Brood, K. 1979. Conulariids. In: V. Jaanusson; S. Laufeld, \& R. Skoglund (eds.) Lower Wenlock faunal and floral dynamics-Vattenfallet section, Gotland, Sveriges Geologiska Undersökning, p. 183-184.

Buitrón, B.E.; Flores de Dios-González, A. \& Vachard, D. 2000. Nuevos hallazgos de invertebrados paleozoicos (ScyphozoaConulata, Archaeogastropoda-Trochina y EchinodermataCrinoidea) en la región de Nochixtlán-Ixtaltepec, Oaxaca. Baja California, Unión Geofísica Mexicana, 298 p. (Boletín 20).

Cloud, P.E. 1944. Permian brachiopods. In: R.E. King; C. Dunbar; P.E. Cloud \& A.K. Miller (eds.) Geology and paleontology of the Permian area northwest of Las Delicias, southwestern Coahuila, Mexico, Boulder, The Geological Society of America, p. 49-69 (Special Paper 52).

Collins, A.G. 2002. Phylogeny of Medusozoa and evolution of cnidarian life cycles. Journal of Evolutionary Biology, 15:418432. doi:10.1046/j.1420-9101.2002.00403.x

Dunbar, C.O. 1944. Permian and Pennsylvanian (?) fusulines. In: R.E King; C.O. Dunbar; P.E. Cloud \& A.K. Miller (eds.) Geology and paleontology of the Permian area northwest of Las Delicias, southwestern Coahuila, Mexico, Boulder, The Geological Society of America, p. 35-48 (Special Paper 52).

Escalante-Ruiz, A.R.; Quiroz-Barroso, S.A. \& Sour-Tovar, F. 2014. Conuláridos Misisípicos de Nochixtlán, Oaxaca, México: consideraciones sistemáticas, estratigráficas y paleobiogeográficas. Revista Brasileira de Paleontologia, 17:195-206. doi:10.4072/rbp.2014.2.07

Finks, R.M. 1955. Conularia in a sponge from the west Texas Permian. Journal of Paleontology, 29:831-836. 
Fletcher, H.O. 1938. A revision of the Australian Conulariae. Records of the Australian Museum, 20:235-255.

Girty, G.H. 1910. The fauna of the Phosphate beds of the Park City Formation. Reston, United States Geological Survey, 82 p. (Bulletin 436).

González-Arreola, C.; Villaseñor-Martínez, A.B. \& CoronaEsquivel, R. 1994. Permian fauna of the Los Arcos Formation, Municipality of Olinalá, State of Guerrero, Mexico. Revista Mexicana de Ciencias Geológicas, 11:214-221.

Götte, A. 1887. Entwicklungsgeschichte der Aurelia aurita und Cotylorhiza tuberculata. Abhandlungen zur Entwicklungsgeschichte der Tiere. Viertes Heft. Hamburg und Leipzig, Verlag von Leopold Voss, 79 p.

Grunt, T.A. \& Shi, G.R. 1997. A hierarchial framework of Permian global marine biogeography. In: INTERNATIONAL GEOLOGICAL CONGRESS ON PALAEONTOLOGY AND HISTORICAL GEOLOGY, 30, 1997. Proceedings, Utrecht, VSP, p. 2-17.

Haack, W. 1914 (1915). Ueber eine marine Permfauna aus Nordmexiko nebst Bemerkungen über Devon daselbst. Zeitschrift der Deutschen Geologischen Gesellschaft, 66:482504.

Haarmann, E. 1913. Geologische Streifzüge in Coahuila. Zeitschrift der Deutschen Geologischen Gesellschaft, 65:18-47.

Hardland, T.L. \& Pickerill, R.K. 1987. Epizoic Schizocrania sp. from the Ordovician Trenton Group of Quebec, with comment son mode of life of conulariids. Journal of Paleontology, 61:844-849. doi:10.1017/S0022336000029176

Hatscheck, B. 1888. Lehrbuch der Zoologie, eine morphologische Übersicht des Thierreiches zur Einfürhrung in das Stadium dieser Wissenschaft. Jena, Gustav Fischer, 432 p.

Hughes, N.C.; Gunderson, G.O. \& Weedom, M. 2000. Late Cambrian conulariids from Wisconsin and Minnesota. Journal of Paleontology, 74:828-838. doi:10.1666/00223360(2000)074<0828:LCCFWA>2.0.CO;2

Ivanstov, A.Y. \& Fedonkin, M.A. 2002. Conulariid-like fossil from the Vendian of Russia: a metazoan clade across the Proterozoic/Paleozoic Boundary. Palaeontology, 45:1219-1229. doi:10.1111/1475-4983.00283

Jerre, F. 1994. Anatomy and phylogenetic significance of Eoconularia loculata, a conulariid from the Silurian of Gotland. Lethaia, 27:97-109. doi:10.1111/j.1502-3931.1994.tb01562.x

Johnston, R.M. 1887. Contribution to the paleontology of the Upper Palaeozoic rocks of Tasmania. Tasmania, Royal Society of Tasmania, p. 4-18 (Papers and Proceedings1866).

Kelly, W.A. 1936. Geology of the mountains bordering the valleys of Acatita and Las Delicias. Boulder, The Geological Society of America, p. 1009-1038 (Bulletin 47). doi:10.1130/GSAB47-1009

Kiderlen, H. 1937. Die Conularien. Über Bau and Leben der resten Scyphozoa. Neues Jahrbuch für Mineralogie, 77:113-169.

King, R.E. 1934. The Permian of Southwestern Coahuila, Mexico. American Journal of Science, 27:98-112.

King, R.E.; Dunbar, C.O.; Cloud, P.E. \& Miller, A.K. 1944. Geology and paleontology of the Permian area northwest of Las Delicias, Southwestern Coahuila, México. Boulder, The Geological Society of America, 170 p. (Special Papers 52). doi:10.1130/SPE52-p1

Koninck, L.G. 1883. Faune du calcaire Carbonifére de la Belgique. 4e partie, Gastéropodes (suite et fin). Annales du Museé Royal d'Histoire Naturelle de Belgique, 8:1-240.

Leme, J.M.; Rodrigues, S.C.; Simões, M.G. \& Van Iten, H. 2004. Sistemática dos conulários (Cnidaria) da Formação Ponta Grossa
(Devoniano), Estado do Paraná, Brasil. Revista Brasileira de Paleontologia, 7:213-222. doi:10.4072/rbp.2004.2.14

Leme, J.M.; Simões, M.G.; Rodrigues, S.C.; Van Iten, H. \& Marques, A.C. 2008. Cladistic analysis of the suborder Conulariina Miller and Gurley, 1896 (Cnidaria, Scyphozoa; VendianTriassic). Palaeontology, 51:649-662. doi:10.1111/j.14754983.2008.00775.x

Leme, J.M.; Simões, M. \& Van Iten, H. 2010. Phylogenetic sustematics and evolution of conulariids. Systematics and evolution of a problematical fósil taxon: the family Conulariidae (Cnidaria; Ediacaran/Triassic). Saarbrücken, Lambert Academic Publishing, 49 p.

Leme, J.M.; Van Iten, H.; Simões, M.G.; Fairchild, T.R.; Rodrigues, F.; Galante, D. \& Pacheco, M.L.A.F. 2013. A new Ediacaran conulariid from the Tamengo Formation, Corumbá Group, Brazil, and the deep Precambrian evolutionary history of cnidarians. In: CORUMBÁ MEETING, 1, 2013. Abstracts, Corumbá, UnB, p. 15.

Lucas, S.G. 2012. The extinction of the Conulariids. Geosciences, 2:1-10. doi:10.3390/geosciences2010001

McKee, J.W.; Jones, N.W. \& Anderson, T.H. 1999. Late Paleozoic and early Mesozoic history of the Las Delicias terrane, Coahuila, Mexico. In: C. Bartolini; J.L. Wilson; Y T.F. Lawton (eds.) Mesozoic Sedimentary and Tectonic History of North-Central Mexico, Boulder, The Geological Society of America, p. 161-189 (Special Paper 340). doi:10.1130/0-8137-2340-X.161

McKinney, F.K.; Devolvé, J.J. \& Sobieraj, J. 1995. Conularia sp. from the Pyrénnées: further support for scyphozoan affinities of the Conulariida. Lethaia, 28:229-236. doi:10.1111/j.1502-3931.1995. tb01426.x

Miller, A.K. 1944. Geology and paleontology of the Permian area northwest of Las Delicias, Southwestern Coahuila, Mexico: part IV. Permian cephalopods. Boulder, The Geological Society of America, p. 71-128 (Special Paper 52).

Miller, S.A. \& Gurley, W.F.E. 1896. New species of Palaeozoic invertebrates from Illinois and other states. Springfield, Illinois State Museum of Natural History, 50 p. (Bulletin 11).

Moore, R.C. \& Harrington, H. J. 1956. Conulata. In: R.C. Moore (ed.) Treatise on invertebrate paleontology, part $F$, Coelenterata, Geological Society of America, University of Kansas, Lawrence Press, p. 54-66.

Nielsen, C. 2001. Animal evolution: interrelationships of the living Phyla. Oxford, Oxford University Press, 563 p.

Quiroz-Barroso, S.A. \& Perrilliat, M.C. 1997. Pennsylvanian Nuculoids (Bivalvia) from the Ixtaltepec Formation, Oaxaca, Mexico. Journal of Paleontology, 71:400-407. doi:10.1017/ S0022336000039421

Quiroz-Barroso, S.A. \& Sour-Tovar, F. 2006. Fossil record of Upper Paleozoic marine invertebrates from Mexico. In: F.J. Vega; T.G. Nyborg; M.C. Perrilliat; S. Cevallos-Ferriz \& QuirozBarroso, S.A. (eds.) Studies on Mexican paleontolgy, Springer, p. 133-167. doi:10.1007/1-4020-3985-9_8

Rigby, K. \& Senowbari-Daryan, B. 1995. Permian sponge biogeography and biostratigraphy. In: P.A. Scholle; M.P. Tadeusz \& D.S. Ulmer-Scholle (eds.) The Permian of Northern Pangea, Springer-Verlag, p. 153-166. doi:10.1007/978-3-642-785931_10

Rosales-Lagarde, L.; Centeno-García, E.; Dostal, J.; Sour-Tovar, F.; Ochoa-Camarillo, H. \& Quiroz-Barroso, S.A. 2005. The Tuzancoa Formation: evidence of an Early Permian Submarine Continental Arc in East-Central Mexico. International Geology Review, 47:901-919. doi:10.2747/0020-6814.47.9.901 
Sabattini, N. \& Hlebzsevitsh, J.C. 2005. Nueva evidencia sobre el modo de vida de los Conulariida. Ameghiniana, 42:627-632.

Sendino-Lara, M.C. 2009. Revisión de la colección de Conulariidae de The Natural History Museum de Londres. Facultad de Ciencias Geológicas, Universidad Complutense de Madrid, Tesis de Doctorado, $601 \mathrm{p}$.

Sharpe, D. 1856. Descriptions of Paleozoic Mollusca from South Africa. Transactions of the Geological Society of London, 2:206-215.

Shen, S.Z.; Xie, J.F.; Zhang, H. \& Shi, G.R. 2009. Roadian-Wordian (Guadalupian, Middle Permian) global palaeobiogeography of brachiopods. Global and Planetary Change, 65:166-181. doi:10.1016/j.gloplacha.2008.10.017

Shi, G.R. 1995. Tha late Palaeozoic Brachiopod genus Yakovlevia Frefericks, 1925 and the Yakoleviatransversa Zone, northern Yukon Territory, Canada. Proceedings of the Royal Society of Victoria, 107:51-71.

Shi, G.R. \& Grunt, T.A. 2000. Permian Gondwana-Boreal antitropicality with special reference to brachiopod faunas. Palaeogeography, Palaeoclimatology, Palaeoecology, 155:239263. doi:10.1016/S0031-0182(99)00118-2

Sinclair, G.W. 1940. A discussion of the genus Metaconularia with descriptions of new genus. Transactions of the Royal Society of Canada, 34:101-121.

Sinclair, G.W. 1942. A new species of "Conularia” from Gaspé. Le Naturalist Canadien, 69:158-160.

Sinclair, G.W. 1948. The biology of the Conulariida. Mc Grill University, Tesis doctoral, $442 \mathrm{p}$.

Sinclair, G.W. 1952. A classification of the Conularida. FieldanaGeology, 10:135-145.

Sour-Tovar, F. \& Quiroz -Barroso, S.A. 2015. Lower-Middle Permian brachiopods from two localities of Central-South Mexico: faunas of a closing ocean. Permophiles. Newsletter of the Subcommission on Permian Stratigraphy, 61:82-84.

Sour-Tovar, F.; Quiroz-Barroso, S.A.; Quiroz-Barragán, J.; TorresMartínez, M.A. \& González-Mora, S. 2016. Presencia de Anisopyge perannulata y Ditomopyge cf. D. whitei (Trilobita, Proetidae) en el Pérmico (Guadalupiano medio) de la Formación Las Delicias, Coahuila, norte de México. Paleontología Mexicana, 5:103-109.

Sowerby, J. 1821. The mineral conchology of Great Britain; or colored figures and descriptions of those remains of Testaceous animals or shells, which have been preserved at various times and depths in the Earth. The mineral conchology of Great Britain, 3:1-689.

Strimple, H.L. 1971. A Permian Crinoid from Coahuila, Mexico. Journal of Paleontology, 45:1040-1042.

Sugiyama, T. 1942. Studies on the Japanese Conularida. Journal of the Geological Society of Japan, 49:390-399.

Thomas, G.A. 1969. Notoconularia, a new conularid genus from the Permian of eastern Australia. Journal of Paleontology, 43:1283-1290.

Torres-Martínez, M.A.; Heredia-Jiménez, D.P.; Quiroz-Barroso, S.A.; Navas-Parejo, P.; Sour-Tovar, F. \& Quiroz-Barragán, J. 2019. A Permian (late Guadalupian) brachiopod fauna from northeast Mexico and their paleobiogeographic affinities. Journal of South American Earth Sciences, 92:41-55. doi:10.1016/j.jsames.2019.03.002

Van Iten, H. 1991a. Evolutionary affinities of conulariids. In: M.A. Simonetta \& S.C. Morris (eds.) The early evolution of Metazoa and the significance of problematic taxa, Cambridge University Press, p. 145-155.

Van Iten, H. 1991b. Anatomy, patterns of occurrence, and nature of the conulariid schott. Palaeontology, 34:939-954.
Van Iten, H. 1991c. Repositories of and additional comments on Permian sponge specimens containing molds of Paraconularia. Journal of Paleontology, 65:335-337. doi:10.1017/ S002233600002062X

Van Iten, H. 1992a. Morphology and phylogenetic significance of the corners and midlines of the conulariid test. Palaeontology, 35:335-358

Van Iten, H. 1992b. Microstructure and growth of the conulariid test: implications for conulariid affinities. Palaeontology, 35:359-372.

Van Iten, H.; Fitzke, J.A. \& Cox, R.S. 1996. Problematical fossil cnidarians from the Upper Ordovician of the north-central USA. Palaeontology, 39:1037-1064.

Van Iten, H.; Zhi-Kang, Z. \& Mao-Yan, Z. 2000. Anatomy and systematic of the Devonian conulariids Changshaconus Zhu, 1985 and Reticulaconularia Babcock \& Feldmann, 1986. Acta Palaeontologica Sinica, 39:466-475.

von Salvini-Plawen, L. 1978. On the origin and evolution of the lower metazoa. Zeitschriftfur Zoologische Cnidaria und Evolutionsforschung, 16:40-88.

Wade, M. 1994. Scyphozoa fossiles: Vendimedusae, Cubomedusae, Scyphomedusae, Conulata. In: J. Bouillon; C. Carré; D. Carré; A. Franc; J. Goy; M.L. Hernández-Nicais; Y. Tifon, D. Van de Vyer \& M.Wade (eds.) Traité de Zoologie, Tome III, Fascicule 2 - Cnideries et Ctenaires, Masson, p. 923-942.

Walcott, C.D. 1886. Cambrian faunas of North America. Reston, United States Geological Survey, p. 72-89 (Bulletin 30).

Wardlaw, B.R.; Furnish, W.M. \& Nestell, M.K. 1979. Geology and paleontology of the Permian beds near Las Delicias, Coahuila, Mexico. Boulder, The Geological Society of America, p. 111-116 (Bulletin 90). doi:10.1130/0016-7606(1979)90<111:GAPOTP $>2.0 . \mathrm{CO} ; 2$

Waterhouse, J.B. 1979. Permian and Triassic Conulariid species from New Zealand. Journal of the Royal Society of New Zealand, 9:475-489.

Waterhouse, J.B. 1986. Late Paleozoic Scyphozoa and Brachiopoda (Inarticualata, Strophomenida, Productida and Rhynchonellida) from the southeast Bowen Basin, Australia. Palaeontographica Abteilung A, 193:1-91.

Waterhouse, J.B. \& Bonham-Carter, G. 1975. Global distribution and character of Permian biomes base on brachiopod assemblages. Canadian Journal of Earth Sciences, 12:10851146. doi:10.1139/e75-104

Weldon, E.A. \& Shi, G.R. 2003. Global distribution of Permian Conulariids and palaeogeographical implications. Gondwana Research, 6:791-802. doi:10.1016/S1342-937X(05) 71025-X

Werner, B. 1973. New investigations on systematics and evolution of Scyphozoa and the phylum Cnidaria. Woods Hole, Set Marine Biological Laboratory, p. 212-217 (Publications 18).

Worthen, A.H. 1883. Description of some new species of fossil shells from the Lower Carboniferous limestones and Coal Measures of Illinois. Geological Survey of Illinois, 7:323-326.

Yancey, T.E. 1975. Permian marine biotic provinces in North America. Journal of Paleontology, 49:758-766.

Ziegler, A.M.; Hulver, M.L. \& Rowley, D.B. 1997. Permian world topography and climate. In: I.P. Martini (ed.) Late glacial and postglacial environmental changes: Quaternary, CarboniferousPermian and Proterozoic, Oxford University, p. 111-146.

Received in 13 February, 2019; accepted in 21 May, 2019. 